\title{
Reverse Mathematics and the Coloring Number of Graphs
}

\author{
Matthew Jura
}

\begin{abstract}
We use methods of reverse mathematics to analyze the proof theoretic strength of a theorem involving the notion of coloring number. Classically, the coloring number of a graph $G=(V, E)$ is the least cardinal $\kappa$ such that there is a well-ordering of $V$ for which below any vertex in $V$ there are fewer than $\kappa$ many vertices connected to it by $E$. We will study a theorem due to Komjáth and Milner, stating that if a graph is the union of $n$ forests, then the coloring number of the graph is at most $2 n$. We focus on the case when $n=1$.
\end{abstract}

\section{Introduction}

We assume the reader is familiar with the general program of reverse mathematics, in which we study the proof-theoretic strength of theorems of ordinary, "essentially countable" mathematics. For more on reverse mathematics, we refer the reader to Simpson [6]; for background in computability theory, we refer the reader to Soare [7]; for background in graph theory, see Diestel [1]. Within this paper, we will only be working within the subsystems $R C A_{0}, W K L_{0}$, and $A C A_{0}$.

We will use the following lemma from [6] extensively.

Lemma 1.1 (Simpson) The following are pairwise equivalent over $\mathrm{RCA}_{0}$ :

1. $\mathrm{ACA}_{0}$;

2. For all one-to-one functions $f: \mathbb{N} \rightarrow \mathbb{N}$ there exists a set $X \subseteq \mathbb{N}$ such that $(\forall n)[n \in X \leftrightarrow \exists m(f(m)=n)]$; that is, $X$ is the range of $f$.

First we clarify some of the notation used in this paper. Note that within $\mathrm{RCA}_{0}$, every finite set can be encoded as a unique natural number and we denote the set of all codes for finite subsets of $A \subseteq \mathbb{N}$ by Fin ${ }_{A}$. Similarly, every finite sequence can be encoded as a unique natural number, and we denote the set of all codes for finite

Received October 26, 2011; accepted August 9, 2013

First published online September 30, 2015

2010 Mathematics Subject Classification: Primary 03B30, 03D80; Secondary 05C15

Keywords: reverse mathematics, coloring number, graph, computability theory

(C) 2016 by University of Notre Dame 10.1215/00294527-3321905 


\title{
Reverse Mathematics and the Coloring Number of Graphs
}

\author{
Matthew Jura
}

\begin{abstract}
We use methods of reverse mathematics to analyze the proof theoretic strength of a theorem involving the notion of coloring number. Classically, the coloring number of a graph $G=(V, E)$ is the least cardinal $\kappa$ such that there is a well-ordering of $V$ for which below any vertex in $V$ there are fewer than $\kappa$ many vertices connected to it by $E$. We will study a theorem due to Komjáth and Milner, stating that if a graph is the union of $n$ forests, then the coloring number of the graph is at most $2 n$. We focus on the case when $n=1$.
\end{abstract}

\section{Introduction}

We assume the reader is familiar with the general program of reverse mathematics, in which we study the proof-theoretic strength of theorems of ordinary, "essentially countable" mathematics. For more on reverse mathematics, we refer the reader to Simpson [6]; for background in computability theory, we refer the reader to Soare [7]; for background in graph theory, see Diestel [1]. Within this paper, we will only be working within the subsystems $R C A_{0}, W K L_{0}$, and $A C A_{0}$.

We will use the following lemma from [6] extensively.

Lemma 1.1 (Simpson) The following are pairwise equivalent over $\mathrm{RCA}_{0}$ :

1. $\mathrm{ACA}_{0}$;

2. For all one-to-one functions $f: \mathbb{N} \rightarrow \mathbb{N}$ there exists a set $X \subseteq \mathbb{N}$ such that $(\forall n)[n \in X \leftrightarrow \exists m(f(m)=n)]$; that is, $X$ is the range of $f$.

First we clarify some of the notation used in this paper. Note that within $\mathrm{RCA}_{0}$, every finite set can be encoded as a unique natural number and we denote the set of all codes for finite subsets of $A \subseteq \mathbb{N}$ by Fin ${ }_{A}$. Similarly, every finite sequence can be encoded as a unique natural number, and we denote the set of all codes for finite

Received October 26, 2011; accepted August 9, 2013

First published online September 30, 2015

2010 Mathematics Subject Classification: Primary 03B30, 03D80; Secondary 05C15

Keywords: reverse mathematics, coloring number, graph, computability theory

(C) 2016 by University of Notre Dame 10.1215/00294527-3321905 


\title{
Reverse Mathematics and the Coloring Number of Graphs
}

\author{
Matthew Jura
}

\begin{abstract}
We use methods of reverse mathematics to analyze the proof theoretic strength of a theorem involving the notion of coloring number. Classically, the coloring number of a graph $G=(V, E)$ is the least cardinal $\kappa$ such that there is a well-ordering of $V$ for which below any vertex in $V$ there are fewer than $\kappa$ many vertices connected to it by $E$. We will study a theorem due to Komjáth and Milner, stating that if a graph is the union of $n$ forests, then the coloring number of the graph is at most $2 n$. We focus on the case when $n=1$.
\end{abstract}

\section{Introduction}

We assume the reader is familiar with the general program of reverse mathematics, in which we study the proof-theoretic strength of theorems of ordinary, "essentially countable" mathematics. For more on reverse mathematics, we refer the reader to Simpson [6]; for background in computability theory, we refer the reader to Soare [7]; for background in graph theory, see Diestel [1]. Within this paper, we will only be working within the subsystems $R C A_{0}, W K L_{0}$, and $A C A_{0}$.

We will use the following lemma from [6] extensively.

Lemma 1.1 (Simpson) The following are pairwise equivalent over $\mathrm{RCA}_{0}$ :

1. $\mathrm{ACA}_{0}$;

2. For all one-to-one functions $f: \mathbb{N} \rightarrow \mathbb{N}$ there exists a set $X \subseteq \mathbb{N}$ such that $(\forall n)[n \in X \leftrightarrow \exists m(f(m)=n)]$; that is, $X$ is the range of $f$.

First we clarify some of the notation used in this paper. Note that within $\mathrm{RCA}_{0}$, every finite set can be encoded as a unique natural number and we denote the set of all codes for finite subsets of $A \subseteq \mathbb{N}$ by Fin ${ }_{A}$. Similarly, every finite sequence can be encoded as a unique natural number, and we denote the set of all codes for finite

Received October 26, 2011; accepted August 9, 2013

First published online September 30, 2015

2010 Mathematics Subject Classification: Primary 03B30, 03D80; Secondary 05C15

Keywords: reverse mathematics, coloring number, graph, computability theory

(C) 2016 by University of Notre Dame 10.1215/00294527-3321905 


\title{
Reverse Mathematics and the Coloring Number of Graphs
}

\author{
Matthew Jura
}

\begin{abstract}
We use methods of reverse mathematics to analyze the proof theoretic strength of a theorem involving the notion of coloring number. Classically, the coloring number of a graph $G=(V, E)$ is the least cardinal $\kappa$ such that there is a well-ordering of $V$ for which below any vertex in $V$ there are fewer than $\kappa$ many vertices connected to it by $E$. We will study a theorem due to Komjáth and Milner, stating that if a graph is the union of $n$ forests, then the coloring number of the graph is at most $2 n$. We focus on the case when $n=1$.
\end{abstract}

\section{Introduction}

We assume the reader is familiar with the general program of reverse mathematics, in which we study the proof-theoretic strength of theorems of ordinary, "essentially countable" mathematics. For more on reverse mathematics, we refer the reader to Simpson [6]; for background in computability theory, we refer the reader to Soare [7]; for background in graph theory, see Diestel [1]. Within this paper, we will only be working within the subsystems $R C A_{0}, W K L_{0}$, and $A C A_{0}$.

We will use the following lemma from [6] extensively.

Lemma 1.1 (Simpson) The following are pairwise equivalent over $\mathrm{RCA}_{0}$ :

1. $\mathrm{ACA}_{0}$;

2. For all one-to-one functions $f: \mathbb{N} \rightarrow \mathbb{N}$ there exists a set $X \subseteq \mathbb{N}$ such that $(\forall n)[n \in X \leftrightarrow \exists m(f(m)=n)]$; that is, $X$ is the range of $f$.

First we clarify some of the notation used in this paper. Note that within $\mathrm{RCA}_{0}$, every finite set can be encoded as a unique natural number and we denote the set of all codes for finite subsets of $A \subseteq \mathbb{N}$ by Fin ${ }_{A}$. Similarly, every finite sequence can be encoded as a unique natural number, and we denote the set of all codes for finite

Received October 26, 2011; accepted August 9, 2013

First published online September 30, 2015

2010 Mathematics Subject Classification: Primary 03B30, 03D80; Secondary 05C15

Keywords: reverse mathematics, coloring number, graph, computability theory

(C) 2016 by University of Notre Dame 10.1215/00294527-3321905 


\title{
Reverse Mathematics and the Coloring Number of Graphs
}

\author{
Matthew Jura
}

\begin{abstract}
We use methods of reverse mathematics to analyze the proof theoretic strength of a theorem involving the notion of coloring number. Classically, the coloring number of a graph $G=(V, E)$ is the least cardinal $\kappa$ such that there is a well-ordering of $V$ for which below any vertex in $V$ there are fewer than $\kappa$ many vertices connected to it by $E$. We will study a theorem due to Komjáth and Milner, stating that if a graph is the union of $n$ forests, then the coloring number of the graph is at most $2 n$. We focus on the case when $n=1$.
\end{abstract}

\section{Introduction}

We assume the reader is familiar with the general program of reverse mathematics, in which we study the proof-theoretic strength of theorems of ordinary, "essentially countable" mathematics. For more on reverse mathematics, we refer the reader to Simpson [6]; for background in computability theory, we refer the reader to Soare [7]; for background in graph theory, see Diestel [1]. Within this paper, we will only be working within the subsystems $R C A_{0}, W K L_{0}$, and $A C A_{0}$.

We will use the following lemma from [6] extensively.

Lemma 1.1 (Simpson) The following are pairwise equivalent over $\mathrm{RCA}_{0}$ :

1. $\mathrm{ACA}_{0}$;

2. For all one-to-one functions $f: \mathbb{N} \rightarrow \mathbb{N}$ there exists a set $X \subseteq \mathbb{N}$ such that $(\forall n)[n \in X \leftrightarrow \exists m(f(m)=n)]$; that is, $X$ is the range of $f$.

First we clarify some of the notation used in this paper. Note that within $\mathrm{RCA}_{0}$, every finite set can be encoded as a unique natural number and we denote the set of all codes for finite subsets of $A \subseteq \mathbb{N}$ by Fin ${ }_{A}$. Similarly, every finite sequence can be encoded as a unique natural number, and we denote the set of all codes for finite

Received October 26, 2011; accepted August 9, 2013

First published online September 30, 2015

2010 Mathematics Subject Classification: Primary 03B30, 03D80; Secondary 05C15

Keywords: reverse mathematics, coloring number, graph, computability theory

(C) 2016 by University of Notre Dame 10.1215/00294527-3321905 


\title{
Reverse Mathematics and the Coloring Number of Graphs
}

\author{
Matthew Jura
}

\begin{abstract}
We use methods of reverse mathematics to analyze the proof theoretic strength of a theorem involving the notion of coloring number. Classically, the coloring number of a graph $G=(V, E)$ is the least cardinal $\kappa$ such that there is a well-ordering of $V$ for which below any vertex in $V$ there are fewer than $\kappa$ many vertices connected to it by $E$. We will study a theorem due to Komjáth and Milner, stating that if a graph is the union of $n$ forests, then the coloring number of the graph is at most $2 n$. We focus on the case when $n=1$.
\end{abstract}

\section{Introduction}

We assume the reader is familiar with the general program of reverse mathematics, in which we study the proof-theoretic strength of theorems of ordinary, "essentially countable" mathematics. For more on reverse mathematics, we refer the reader to Simpson [6]; for background in computability theory, we refer the reader to Soare [7]; for background in graph theory, see Diestel [1]. Within this paper, we will only be working within the subsystems $R C A_{0}, W K L_{0}$, and $A C A_{0}$.

We will use the following lemma from [6] extensively.

Lemma 1.1 (Simpson) The following are pairwise equivalent over $\mathrm{RCA}_{0}$ :

1. $\mathrm{ACA}_{0}$;

2. For all one-to-one functions $f: \mathbb{N} \rightarrow \mathbb{N}$ there exists a set $X \subseteq \mathbb{N}$ such that $(\forall n)[n \in X \leftrightarrow \exists m(f(m)=n)]$; that is, $X$ is the range of $f$.

First we clarify some of the notation used in this paper. Note that within $\mathrm{RCA}_{0}$, every finite set can be encoded as a unique natural number and we denote the set of all codes for finite subsets of $A \subseteq \mathbb{N}$ by Fin ${ }_{A}$. Similarly, every finite sequence can be encoded as a unique natural number, and we denote the set of all codes for finite

Received October 26, 2011; accepted August 9, 2013

First published online September 30, 2015

2010 Mathematics Subject Classification: Primary 03B30, 03D80; Secondary 05C15

Keywords: reverse mathematics, coloring number, graph, computability theory

(C) 2016 by University of Notre Dame 10.1215/00294527-3321905 


\title{
Reverse Mathematics and the Coloring Number of Graphs
}

\author{
Matthew Jura
}

\begin{abstract}
We use methods of reverse mathematics to analyze the proof theoretic strength of a theorem involving the notion of coloring number. Classically, the coloring number of a graph $G=(V, E)$ is the least cardinal $\kappa$ such that there is a well-ordering of $V$ for which below any vertex in $V$ there are fewer than $\kappa$ many vertices connected to it by $E$. We will study a theorem due to Komjáth and Milner, stating that if a graph is the union of $n$ forests, then the coloring number of the graph is at most $2 n$. We focus on the case when $n=1$.
\end{abstract}

\section{Introduction}

We assume the reader is familiar with the general program of reverse mathematics, in which we study the proof-theoretic strength of theorems of ordinary, "essentially countable" mathematics. For more on reverse mathematics, we refer the reader to Simpson [6]; for background in computability theory, we refer the reader to Soare [7]; for background in graph theory, see Diestel [1]. Within this paper, we will only be working within the subsystems $R C A_{0}, W K L_{0}$, and $A C A_{0}$.

We will use the following lemma from [6] extensively.

Lemma 1.1 (Simpson) The following are pairwise equivalent over $\mathrm{RCA}_{0}$ :

1. $\mathrm{ACA}_{0}$;

2. For all one-to-one functions $f: \mathbb{N} \rightarrow \mathbb{N}$ there exists a set $X \subseteq \mathbb{N}$ such that $(\forall n)[n \in X \leftrightarrow \exists m(f(m)=n)]$; that is, $X$ is the range of $f$.

First we clarify some of the notation used in this paper. Note that within $\mathrm{RCA}_{0}$, every finite set can be encoded as a unique natural number and we denote the set of all codes for finite subsets of $A \subseteq \mathbb{N}$ by Fin ${ }_{A}$. Similarly, every finite sequence can be encoded as a unique natural number, and we denote the set of all codes for finite

Received October 26, 2011; accepted August 9, 2013

First published online September 30, 2015

2010 Mathematics Subject Classification: Primary 03B30, 03D80; Secondary 05C15

Keywords: reverse mathematics, coloring number, graph, computability theory

(C) 2016 by University of Notre Dame 10.1215/00294527-3321905 


\title{
Reverse Mathematics and the Coloring Number of Graphs
}

\author{
Matthew Jura
}

\begin{abstract}
We use methods of reverse mathematics to analyze the proof theoretic strength of a theorem involving the notion of coloring number. Classically, the coloring number of a graph $G=(V, E)$ is the least cardinal $\kappa$ such that there is a well-ordering of $V$ for which below any vertex in $V$ there are fewer than $\kappa$ many vertices connected to it by $E$. We will study a theorem due to Komjáth and Milner, stating that if a graph is the union of $n$ forests, then the coloring number of the graph is at most $2 n$. We focus on the case when $n=1$.
\end{abstract}

\section{Introduction}

We assume the reader is familiar with the general program of reverse mathematics, in which we study the proof-theoretic strength of theorems of ordinary, "essentially countable" mathematics. For more on reverse mathematics, we refer the reader to Simpson [6]; for background in computability theory, we refer the reader to Soare [7]; for background in graph theory, see Diestel [1]. Within this paper, we will only be working within the subsystems $R C A_{0}, W K L_{0}$, and $A C A_{0}$.

We will use the following lemma from [6] extensively.

Lemma 1.1 (Simpson) The following are pairwise equivalent over $\mathrm{RCA}_{0}$ :

1. $\mathrm{ACA}_{0}$;

2. For all one-to-one functions $f: \mathbb{N} \rightarrow \mathbb{N}$ there exists a set $X \subseteq \mathbb{N}$ such that $(\forall n)[n \in X \leftrightarrow \exists m(f(m)=n)]$; that is, $X$ is the range of $f$.

First we clarify some of the notation used in this paper. Note that within $\mathrm{RCA}_{0}$, every finite set can be encoded as a unique natural number and we denote the set of all codes for finite subsets of $A \subseteq \mathbb{N}$ by Fin ${ }_{A}$. Similarly, every finite sequence can be encoded as a unique natural number, and we denote the set of all codes for finite

Received October 26, 2011; accepted August 9, 2013

First published online September 30, 2015

2010 Mathematics Subject Classification: Primary 03B30, 03D80; Secondary 05C15

Keywords: reverse mathematics, coloring number, graph, computability theory

(C) 2016 by University of Notre Dame 10.1215/00294527-3321905 


\title{
Reverse Mathematics and the Coloring Number of Graphs
}

\author{
Matthew Jura
}

\begin{abstract}
We use methods of reverse mathematics to analyze the proof theoretic strength of a theorem involving the notion of coloring number. Classically, the coloring number of a graph $G=(V, E)$ is the least cardinal $\kappa$ such that there is a well-ordering of $V$ for which below any vertex in $V$ there are fewer than $\kappa$ many vertices connected to it by $E$. We will study a theorem due to Komjáth and Milner, stating that if a graph is the union of $n$ forests, then the coloring number of the graph is at most $2 n$. We focus on the case when $n=1$.
\end{abstract}

\section{Introduction}

We assume the reader is familiar with the general program of reverse mathematics, in which we study the proof-theoretic strength of theorems of ordinary, "essentially countable" mathematics. For more on reverse mathematics, we refer the reader to Simpson [6]; for background in computability theory, we refer the reader to Soare [7]; for background in graph theory, see Diestel [1]. Within this paper, we will only be working within the subsystems $R C A_{0}, W K L_{0}$, and $A C A_{0}$.

We will use the following lemma from [6] extensively.

Lemma 1.1 (Simpson) The following are pairwise equivalent over $\mathrm{RCA}_{0}$ :

1. $\mathrm{ACA}_{0}$;

2. For all one-to-one functions $f: \mathbb{N} \rightarrow \mathbb{N}$ there exists a set $X \subseteq \mathbb{N}$ such that $(\forall n)[n \in X \leftrightarrow \exists m(f(m)=n)]$; that is, $X$ is the range of $f$.

First we clarify some of the notation used in this paper. Note that within $\mathrm{RCA}_{0}$, every finite set can be encoded as a unique natural number and we denote the set of all codes for finite subsets of $A \subseteq \mathbb{N}$ by Fin ${ }_{A}$. Similarly, every finite sequence can be encoded as a unique natural number, and we denote the set of all codes for finite

Received October 26, 2011; accepted August 9, 2013

First published online September 30, 2015

2010 Mathematics Subject Classification: Primary 03B30, 03D80; Secondary 05C15

Keywords: reverse mathematics, coloring number, graph, computability theory

(C) 2016 by University of Notre Dame 10.1215/00294527-3321905 


\title{
Reverse Mathematics and the Coloring Number of Graphs
}

\author{
Matthew Jura
}

\begin{abstract}
We use methods of reverse mathematics to analyze the proof theoretic strength of a theorem involving the notion of coloring number. Classically, the coloring number of a graph $G=(V, E)$ is the least cardinal $\kappa$ such that there is a well-ordering of $V$ for which below any vertex in $V$ there are fewer than $\kappa$ many vertices connected to it by $E$. We will study a theorem due to Komjáth and Milner, stating that if a graph is the union of $n$ forests, then the coloring number of the graph is at most $2 n$. We focus on the case when $n=1$.
\end{abstract}

\section{Introduction}

We assume the reader is familiar with the general program of reverse mathematics, in which we study the proof-theoretic strength of theorems of ordinary, "essentially countable" mathematics. For more on reverse mathematics, we refer the reader to Simpson [6]; for background in computability theory, we refer the reader to Soare [7]; for background in graph theory, see Diestel [1]. Within this paper, we will only be working within the subsystems $R C A_{0}, W K L_{0}$, and $A C A_{0}$.

We will use the following lemma from [6] extensively.

Lemma 1.1 (Simpson) The following are pairwise equivalent over $\mathrm{RCA}_{0}$ :

1. $\mathrm{ACA}_{0}$;

2. For all one-to-one functions $f: \mathbb{N} \rightarrow \mathbb{N}$ there exists a set $X \subseteq \mathbb{N}$ such that $(\forall n)[n \in X \leftrightarrow \exists m(f(m)=n)]$; that is, $X$ is the range of $f$.

First we clarify some of the notation used in this paper. Note that within $\mathrm{RCA}_{0}$, every finite set can be encoded as a unique natural number and we denote the set of all codes for finite subsets of $A \subseteq \mathbb{N}$ by Fin ${ }_{A}$. Similarly, every finite sequence can be encoded as a unique natural number, and we denote the set of all codes for finite

Received October 26, 2011; accepted August 9, 2013

First published online September 30, 2015

2010 Mathematics Subject Classification: Primary 03B30, 03D80; Secondary 05C15

Keywords: reverse mathematics, coloring number, graph, computability theory

(C) 2016 by University of Notre Dame 10.1215/00294527-3321905 


\title{
Reverse Mathematics and the Coloring Number of Graphs
}

\author{
Matthew Jura
}

\begin{abstract}
We use methods of reverse mathematics to analyze the proof theoretic strength of a theorem involving the notion of coloring number. Classically, the coloring number of a graph $G=(V, E)$ is the least cardinal $\kappa$ such that there is a well-ordering of $V$ for which below any vertex in $V$ there are fewer than $\kappa$ many vertices connected to it by $E$. We will study a theorem due to Komjáth and Milner, stating that if a graph is the union of $n$ forests, then the coloring number of the graph is at most $2 n$. We focus on the case when $n=1$.
\end{abstract}

\section{Introduction}

We assume the reader is familiar with the general program of reverse mathematics, in which we study the proof-theoretic strength of theorems of ordinary, "essentially countable" mathematics. For more on reverse mathematics, we refer the reader to Simpson [6]; for background in computability theory, we refer the reader to Soare [7]; for background in graph theory, see Diestel [1]. Within this paper, we will only be working within the subsystems $R C A_{0}, W K L_{0}$, and $A C A_{0}$.

We will use the following lemma from [6] extensively.

Lemma 1.1 (Simpson) The following are pairwise equivalent over $\mathrm{RCA}_{0}$ :

1. $\mathrm{ACA}_{0}$;

2. For all one-to-one functions $f: \mathbb{N} \rightarrow \mathbb{N}$ there exists a set $X \subseteq \mathbb{N}$ such that $(\forall n)[n \in X \leftrightarrow \exists m(f(m)=n)]$; that is, $X$ is the range of $f$.

First we clarify some of the notation used in this paper. Note that within $\mathrm{RCA}_{0}$, every finite set can be encoded as a unique natural number and we denote the set of all codes for finite subsets of $A \subseteq \mathbb{N}$ by Fin ${ }_{A}$. Similarly, every finite sequence can be encoded as a unique natural number, and we denote the set of all codes for finite

Received October 26, 2011; accepted August 9, 2013

First published online September 30, 2015

2010 Mathematics Subject Classification: Primary 03B30, 03D80; Secondary 05C15

Keywords: reverse mathematics, coloring number, graph, computability theory

(C) 2016 by University of Notre Dame 10.1215/00294527-3321905 


\title{
Reverse Mathematics and the Coloring Number of Graphs
}

\author{
Matthew Jura
}

\begin{abstract}
We use methods of reverse mathematics to analyze the proof theoretic strength of a theorem involving the notion of coloring number. Classically, the coloring number of a graph $G=(V, E)$ is the least cardinal $\kappa$ such that there is a well-ordering of $V$ for which below any vertex in $V$ there are fewer than $\kappa$ many vertices connected to it by $E$. We will study a theorem due to Komjáth and Milner, stating that if a graph is the union of $n$ forests, then the coloring number of the graph is at most $2 n$. We focus on the case when $n=1$.
\end{abstract}

\section{Introduction}

We assume the reader is familiar with the general program of reverse mathematics, in which we study the proof-theoretic strength of theorems of ordinary, "essentially countable" mathematics. For more on reverse mathematics, we refer the reader to Simpson [6]; for background in computability theory, we refer the reader to Soare [7]; for background in graph theory, see Diestel [1]. Within this paper, we will only be working within the subsystems $R C A_{0}, W K L_{0}$, and $A C A_{0}$.

We will use the following lemma from [6] extensively.

Lemma 1.1 (Simpson) The following are pairwise equivalent over $\mathrm{RCA}_{0}$ :

1. $\mathrm{ACA}_{0}$;

2. For all one-to-one functions $f: \mathbb{N} \rightarrow \mathbb{N}$ there exists a set $X \subseteq \mathbb{N}$ such that $(\forall n)[n \in X \leftrightarrow \exists m(f(m)=n)]$; that is, $X$ is the range of $f$.

First we clarify some of the notation used in this paper. Note that within $\mathrm{RCA}_{0}$, every finite set can be encoded as a unique natural number and we denote the set of all codes for finite subsets of $A \subseteq \mathbb{N}$ by Fin ${ }_{A}$. Similarly, every finite sequence can be encoded as a unique natural number, and we denote the set of all codes for finite

Received October 26, 2011; accepted August 9, 2013

First published online September 30, 2015

2010 Mathematics Subject Classification: Primary 03B30, 03D80; Secondary 05C15

Keywords: reverse mathematics, coloring number, graph, computability theory

(C) 2016 by University of Notre Dame 10.1215/00294527-3321905 


\title{
Reverse Mathematics and the Coloring Number of Graphs
}

\author{
Matthew Jura
}

\begin{abstract}
We use methods of reverse mathematics to analyze the proof theoretic strength of a theorem involving the notion of coloring number. Classically, the coloring number of a graph $G=(V, E)$ is the least cardinal $\kappa$ such that there is a well-ordering of $V$ for which below any vertex in $V$ there are fewer than $\kappa$ many vertices connected to it by $E$. We will study a theorem due to Komjáth and Milner, stating that if a graph is the union of $n$ forests, then the coloring number of the graph is at most $2 n$. We focus on the case when $n=1$.
\end{abstract}

\section{Introduction}

We assume the reader is familiar with the general program of reverse mathematics, in which we study the proof-theoretic strength of theorems of ordinary, "essentially countable" mathematics. For more on reverse mathematics, we refer the reader to Simpson [6]; for background in computability theory, we refer the reader to Soare [7]; for background in graph theory, see Diestel [1]. Within this paper, we will only be working within the subsystems $R C A_{0}, W K L_{0}$, and $A C A_{0}$.

We will use the following lemma from [6] extensively.

Lemma 1.1 (Simpson) The following are pairwise equivalent over $\mathrm{RCA}_{0}$ :

1. $\mathrm{ACA}_{0}$;

2. For all one-to-one functions $f: \mathbb{N} \rightarrow \mathbb{N}$ there exists a set $X \subseteq \mathbb{N}$ such that $(\forall n)[n \in X \leftrightarrow \exists m(f(m)=n)]$; that is, $X$ is the range of $f$.

First we clarify some of the notation used in this paper. Note that within $\mathrm{RCA}_{0}$, every finite set can be encoded as a unique natural number and we denote the set of all codes for finite subsets of $A \subseteq \mathbb{N}$ by Fin ${ }_{A}$. Similarly, every finite sequence can be encoded as a unique natural number, and we denote the set of all codes for finite

Received October 26, 2011; accepted August 9, 2013

First published online September 30, 2015

2010 Mathematics Subject Classification: Primary 03B30, 03D80; Secondary 05C15

Keywords: reverse mathematics, coloring number, graph, computability theory

(C) 2016 by University of Notre Dame 10.1215/00294527-3321905 


\title{
Reverse Mathematics and the Coloring Number of Graphs
}

\author{
Matthew Jura
}

\begin{abstract}
We use methods of reverse mathematics to analyze the proof theoretic strength of a theorem involving the notion of coloring number. Classically, the coloring number of a graph $G=(V, E)$ is the least cardinal $\kappa$ such that there is a well-ordering of $V$ for which below any vertex in $V$ there are fewer than $\kappa$ many vertices connected to it by $E$. We will study a theorem due to Komjáth and Milner, stating that if a graph is the union of $n$ forests, then the coloring number of the graph is at most $2 n$. We focus on the case when $n=1$.
\end{abstract}

\section{Introduction}

We assume the reader is familiar with the general program of reverse mathematics, in which we study the proof-theoretic strength of theorems of ordinary, "essentially countable" mathematics. For more on reverse mathematics, we refer the reader to Simpson [6]; for background in computability theory, we refer the reader to Soare [7]; for background in graph theory, see Diestel [1]. Within this paper, we will only be working within the subsystems $R C A_{0}, W K L_{0}$, and $A C A_{0}$.

We will use the following lemma from [6] extensively.

Lemma 1.1 (Simpson) The following are pairwise equivalent over $\mathrm{RCA}_{0}$ :

1. $\mathrm{ACA}_{0}$;

2. For all one-to-one functions $f: \mathbb{N} \rightarrow \mathbb{N}$ there exists a set $X \subseteq \mathbb{N}$ such that $(\forall n)[n \in X \leftrightarrow \exists m(f(m)=n)]$; that is, $X$ is the range of $f$.

First we clarify some of the notation used in this paper. Note that within $\mathrm{RCA}_{0}$, every finite set can be encoded as a unique natural number and we denote the set of all codes for finite subsets of $A \subseteq \mathbb{N}$ by Fin ${ }_{A}$. Similarly, every finite sequence can be encoded as a unique natural number, and we denote the set of all codes for finite

Received October 26, 2011; accepted August 9, 2013

First published online September 30, 2015

2010 Mathematics Subject Classification: Primary 03B30, 03D80; Secondary 05C15

Keywords: reverse mathematics, coloring number, graph, computability theory

(C) 2016 by University of Notre Dame 10.1215/00294527-3321905 


\title{
Reverse Mathematics and the Coloring Number of Graphs
}

\author{
Matthew Jura
}

\begin{abstract}
We use methods of reverse mathematics to analyze the proof theoretic strength of a theorem involving the notion of coloring number. Classically, the coloring number of a graph $G=(V, E)$ is the least cardinal $\kappa$ such that there is a well-ordering of $V$ for which below any vertex in $V$ there are fewer than $\kappa$ many vertices connected to it by $E$. We will study a theorem due to Komjáth and Milner, stating that if a graph is the union of $n$ forests, then the coloring number of the graph is at most $2 n$. We focus on the case when $n=1$.
\end{abstract}

\section{Introduction}

We assume the reader is familiar with the general program of reverse mathematics, in which we study the proof-theoretic strength of theorems of ordinary, "essentially countable" mathematics. For more on reverse mathematics, we refer the reader to Simpson [6]; for background in computability theory, we refer the reader to Soare [7]; for background in graph theory, see Diestel [1]. Within this paper, we will only be working within the subsystems $R C A_{0}, W K L_{0}$, and $A C A_{0}$.

We will use the following lemma from [6] extensively.

Lemma 1.1 (Simpson) The following are pairwise equivalent over $\mathrm{RCA}_{0}$ :

1. $\mathrm{ACA}_{0}$;

2. For all one-to-one functions $f: \mathbb{N} \rightarrow \mathbb{N}$ there exists a set $X \subseteq \mathbb{N}$ such that $(\forall n)[n \in X \leftrightarrow \exists m(f(m)=n)]$; that is, $X$ is the range of $f$.

First we clarify some of the notation used in this paper. Note that within $\mathrm{RCA}_{0}$, every finite set can be encoded as a unique natural number and we denote the set of all codes for finite subsets of $A \subseteq \mathbb{N}$ by Fin ${ }_{A}$. Similarly, every finite sequence can be encoded as a unique natural number, and we denote the set of all codes for finite

Received October 26, 2011; accepted August 9, 2013

First published online September 30, 2015

2010 Mathematics Subject Classification: Primary 03B30, 03D80; Secondary 05C15

Keywords: reverse mathematics, coloring number, graph, computability theory

(C) 2016 by University of Notre Dame 10.1215/00294527-3321905 


\title{
Reverse Mathematics and the Coloring Number of Graphs
}

\author{
Matthew Jura
}

\begin{abstract}
We use methods of reverse mathematics to analyze the proof theoretic strength of a theorem involving the notion of coloring number. Classically, the coloring number of a graph $G=(V, E)$ is the least cardinal $\kappa$ such that there is a well-ordering of $V$ for which below any vertex in $V$ there are fewer than $\kappa$ many vertices connected to it by $E$. We will study a theorem due to Komjáth and Milner, stating that if a graph is the union of $n$ forests, then the coloring number of the graph is at most $2 n$. We focus on the case when $n=1$.
\end{abstract}

\section{Introduction}

We assume the reader is familiar with the general program of reverse mathematics, in which we study the proof-theoretic strength of theorems of ordinary, "essentially countable" mathematics. For more on reverse mathematics, we refer the reader to Simpson [6]; for background in computability theory, we refer the reader to Soare [7]; for background in graph theory, see Diestel [1]. Within this paper, we will only be working within the subsystems $R C A_{0}, W K L_{0}$, and $A C A_{0}$.

We will use the following lemma from [6] extensively.

Lemma 1.1 (Simpson) The following are pairwise equivalent over $\mathrm{RCA}_{0}$ :

1. $\mathrm{ACA}_{0}$;

2. For all one-to-one functions $f: \mathbb{N} \rightarrow \mathbb{N}$ there exists a set $X \subseteq \mathbb{N}$ such that $(\forall n)[n \in X \leftrightarrow \exists m(f(m)=n)]$; that is, $X$ is the range of $f$.

First we clarify some of the notation used in this paper. Note that within $\mathrm{RCA}_{0}$, every finite set can be encoded as a unique natural number and we denote the set of all codes for finite subsets of $A \subseteq \mathbb{N}$ by Fin ${ }_{A}$. Similarly, every finite sequence can be encoded as a unique natural number, and we denote the set of all codes for finite

Received October 26, 2011; accepted August 9, 2013

First published online September 30, 2015

2010 Mathematics Subject Classification: Primary 03B30, 03D80; Secondary 05C15

Keywords: reverse mathematics, coloring number, graph, computability theory

(C) 2016 by University of Notre Dame 10.1215/00294527-3321905 


\title{
Reverse Mathematics and the Coloring Number of Graphs
}

\author{
Matthew Jura
}

\begin{abstract}
We use methods of reverse mathematics to analyze the proof theoretic strength of a theorem involving the notion of coloring number. Classically, the coloring number of a graph $G=(V, E)$ is the least cardinal $\kappa$ such that there is a well-ordering of $V$ for which below any vertex in $V$ there are fewer than $\kappa$ many vertices connected to it by $E$. We will study a theorem due to Komjáth and Milner, stating that if a graph is the union of $n$ forests, then the coloring number of the graph is at most $2 n$. We focus on the case when $n=1$.
\end{abstract}

\section{Introduction}

We assume the reader is familiar with the general program of reverse mathematics, in which we study the proof-theoretic strength of theorems of ordinary, "essentially countable" mathematics. For more on reverse mathematics, we refer the reader to Simpson [6]; for background in computability theory, we refer the reader to Soare [7]; for background in graph theory, see Diestel [1]. Within this paper, we will only be working within the subsystems $R C A_{0}, W K L_{0}$, and $A C A_{0}$.

We will use the following lemma from [6] extensively.

Lemma 1.1 (Simpson) The following are pairwise equivalent over $\mathrm{RCA}_{0}$ :

1. $\mathrm{ACA}_{0}$;

2. For all one-to-one functions $f: \mathbb{N} \rightarrow \mathbb{N}$ there exists a set $X \subseteq \mathbb{N}$ such that $(\forall n)[n \in X \leftrightarrow \exists m(f(m)=n)]$; that is, $X$ is the range of $f$.

First we clarify some of the notation used in this paper. Note that within $\mathrm{RCA}_{0}$, every finite set can be encoded as a unique natural number and we denote the set of all codes for finite subsets of $A \subseteq \mathbb{N}$ by Fin ${ }_{A}$. Similarly, every finite sequence can be encoded as a unique natural number, and we denote the set of all codes for finite

Received October 26, 2011; accepted August 9, 2013

First published online September 30, 2015

2010 Mathematics Subject Classification: Primary 03B30, 03D80; Secondary 05C15

Keywords: reverse mathematics, coloring number, graph, computability theory

(C) 2016 by University of Notre Dame 10.1215/00294527-3321905 


\title{
Reverse Mathematics and the Coloring Number of Graphs
}

\author{
Matthew Jura
}

\begin{abstract}
We use methods of reverse mathematics to analyze the proof theoretic strength of a theorem involving the notion of coloring number. Classically, the coloring number of a graph $G=(V, E)$ is the least cardinal $\kappa$ such that there is a well-ordering of $V$ for which below any vertex in $V$ there are fewer than $\kappa$ many vertices connected to it by $E$. We will study a theorem due to Komjáth and Milner, stating that if a graph is the union of $n$ forests, then the coloring number of the graph is at most $2 n$. We focus on the case when $n=1$.
\end{abstract}

\section{Introduction}

We assume the reader is familiar with the general program of reverse mathematics, in which we study the proof-theoretic strength of theorems of ordinary, "essentially countable" mathematics. For more on reverse mathematics, we refer the reader to Simpson [6]; for background in computability theory, we refer the reader to Soare [7]; for background in graph theory, see Diestel [1]. Within this paper, we will only be working within the subsystems $R C A_{0}, W K L_{0}$, and $A C A_{0}$.

We will use the following lemma from [6] extensively.

Lemma 1.1 (Simpson) The following are pairwise equivalent over $\mathrm{RCA}_{0}$ :

1. $\mathrm{ACA}_{0}$;

2. For all one-to-one functions $f: \mathbb{N} \rightarrow \mathbb{N}$ there exists a set $X \subseteq \mathbb{N}$ such that $(\forall n)[n \in X \leftrightarrow \exists m(f(m)=n)]$; that is, $X$ is the range of $f$.

First we clarify some of the notation used in this paper. Note that within $\mathrm{RCA}_{0}$, every finite set can be encoded as a unique natural number and we denote the set of all codes for finite subsets of $A \subseteq \mathbb{N}$ by Fin ${ }_{A}$. Similarly, every finite sequence can be encoded as a unique natural number, and we denote the set of all codes for finite

Received October 26, 2011; accepted August 9, 2013

First published online September 30, 2015

2010 Mathematics Subject Classification: Primary 03B30, 03D80; Secondary 05C15

Keywords: reverse mathematics, coloring number, graph, computability theory

(C) 2016 by University of Notre Dame 10.1215/00294527-3321905 